\title{
Characterization of GigaRad Total Ionizing Dose and Annealing Effects on 28-nm Bulk MOSFETs
}

\author{
Chun-Min Zhang, Student Member, IEEE, Farzan Jazaeri, Member, IEEE, Alessandro Pezzotta, \\ Claudio Bruschini, Member, IEEE, Giulio Borghello, Federico Faccio, Serena Mattiazzo, \\ Andrea Baschirotto, Fellow, IEEE, and Christian Enz, Senior Member, IEEE
}

\begin{abstract}
This paper investigates the radiation tolerance of 28-nm bulk $n$ and $p$ MOSFETs up to 1 Grad of total ionizing dose (TID). The radiation effects on this commercial $28-\mathrm{nm}$ bulk CMOS process demonstrate a strong geometry dependence as a result of the complex interplay of oxide and interface charge trapping relevant to the gate-related dielectrics and the shallow trench isolation. The narrowest/longest channel devices have the most serious performance degradation. In addition, $n$ MOSFETs present a limited on-current variation and a significant off-current increase, while $p$ MOSFETs show a negligible off-current change and a substantial on-current degradation. The postirradiation annealing annihilates or neutralizes oxide trapped positive charges and tends to partly recover the degraded device performance. To quantify the effects of TID and postirradiation annealing, parameters including the threshold voltage, the free carrier mobility, the subthreshold swing, and the drain-induced barrier lowering are extracted.
\end{abstract}

Index Terms-28-nm bulk MOSFETs, annealing, charge trapping, high- $k$, interface traps, oxide traps, shallow trench isolation (STI), spacer, total ionizing dose (TID).

\section{INTRODUCTION}

$\mathbf{T}$ $O$ extend the discovery potential at the forefront of research in high-energy physics (HEP), the Large Hadron Collider (LHC) at Conseil Européen pour la Recherche Nucléaire (CERN) will be upgraded for a 10 -fold increase in the integrated luminosity. The high-luminosity LHC (HL-LHC) is expected to experience an unprecedented radiation level up to $1 \mathrm{Grad}$ (10 MGy) of total ionizing

Manuscript received July 11, 2017; revised August 19, 2017; accepted August 20, 2017. Date of publication September 5, 2017; date of current version October 17, 2017. This work was supported in part by the GigaRadMOST project funded by the Swiss National Science Foundation under Grant 200021_160185 and in part by the ScalTech28 project funded by the Istituto Nazionale di Fisica Nucleare.

C.-M. Zhang, F. Jazaeri, A. Pezzotta, C. Bruschini, and C. Enz are with the Integrated Circuits Laboratory, École Polytechnique Fédérale de Lausanne, 2002 Neuchâtel, Switzerland (e-mail: chunmin.zhang@epfl.ch; farzan.jazaeri@epfl.ch; alessandro.pezzotta@epfl.ch; claudio.bruschini@ epfl.ch; christian.enz@epfl.ch).

G. Borghello is with the EP-ESE Group, CERN, 1211 Geneva, Switzerland, and also with the University of Udine, 33100 Udine, Italy (e-mail: giulio.borghello@cern.ch).

F. Faccio is with the EP-ESE Group, CERN, 1211 Geneva, Switzerland (e-mail: federico.faccio@cern.ch).

S. Mattiazzo is with the Department of Information Engineering, University of Padova, 35131 Padova, Italy (e-mail: serena.mattiazzo@dei.unipd.it).

A. Baschirotto is with the University of Milano-Bicocca, 20126 Milan, Italy, and also INFN Milano-Bicocca, 20126 Milan, Italy (e-mail: andrea.baschirotto@unimib.it).

Color versions of one or more of the figures in this paper are available online at http://ieeexplore.ieee.org.

Digital Object Identifier 10.1109/TNS.2017.2746719 dose (TID) and $10^{16}$ neutrons $/ \mathrm{cm}^{2}$ of hadron fluence over 10 years of operation. Hence, the experimental equipments need highly improved radiation-tolerant tracking systems with higher bandwidth and more radiation-tolerant front-end (FE) electronics [1], [2]. The aggressive downscaling of deepsubmicrometer CMOS technologies bring analog and mixedsignal circuit designers the benefits of a higher operation speed and an extended circuit functionality [3]. Furthermore, the gate dielectric with ultrascaled silicon dioxide $\left(\mathrm{SiO}_{2}\right)$ demonstrates a higher radiation tolerance and relevant CMOS technologies are promising for radiation-tolerant applications [4].

Nonetheless, since the device dimensions shrank into 45-nm technology node and beyond, hafnium oxide $\left(\mathrm{HfO}_{2}\right)$ has been increasingly used for a higher physical oxide thickness to resolve the crucial gate direct tunneling issue [5]. However, a physically thicker gate dielectric degrades the radiation tolerance improvement of the aggressive gate-oxide scaling in terms of oxide charge trapping. Considering also the fact that the interface charge trapping relies on the interface quality instead of the oxide thickness, efforts are still needed for investigating the radiation response of the $\mathrm{HfO}_{2}$-based material system [6]-[8]. On the other hand, it is reported in [9] and [10] that the thick spacers severely affect 65-nm short-channel MOSFETs. Especially, the irradiated short-channel $p$ MOSFETs almost degrade to the point of not having any on-current after 1 Grad. Additionally, the shallow trench isolation (STI) oxide is still in the order of $100 \mathrm{~nm}$. The relevant charge buildup imposes strong effects on deepsubmicrometer MOSFETs [11]-[13]. One of the main phenomena is the parasitic sidewall leakage current. Concerning the gate-related dielectrics and the thick STI oxide, it is still of importance to investigate TID effects on ultrascaled MOSFETs for selecting the most appropriate candidates for radiationtolerant circuits in the HL-LHC.

This paper studies DC characteristics of 28-nm bulk MOSFETs up to $1 \mathrm{Grad}$ that is the foreseen radiation level in the innermost detectors of the HL-LHC. We also investigate the electrical evolution of the irradiated devices during annealing. Various sizes of individual MOSFETs are explored for comprehending the dominant mechanisms of TID effects. The results are also helpful for circuit designers to better choose the suitable sizes of MOSFETs for their radiation-tolerant applications. Extending our previous work on $n$ MOSFETs [14], [15], this paper includes a detailed analysis of TID and annealing effects on important parameters 


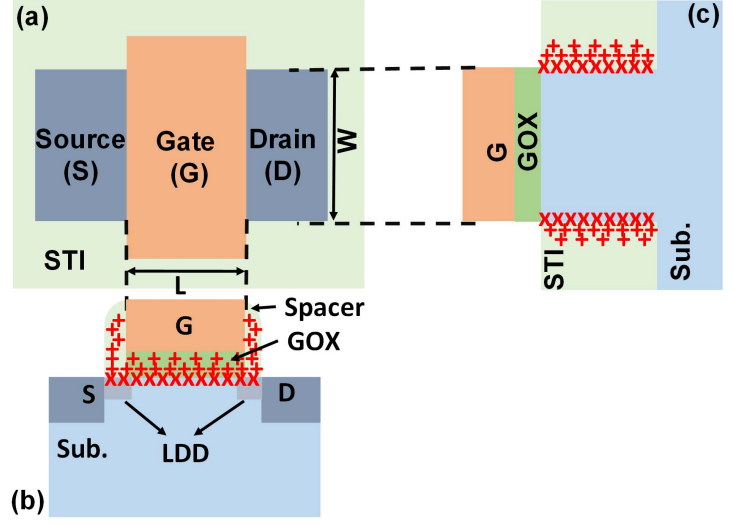

Fig. 1. (a) Top view of a general MOSFET. (b) Cross section along the length of the MOSFET. (c) Cross section along the width of the MOSFET. "+" indicates oxide trapped charges $\left(\mathrm{Q}_{o t}\right)$ and " $\times$ " stands for interface charged traps $\left(\mathrm{Q}_{i t}\right)$.

of 28-nm bulk $n$ and $p$ MOSFETs. The main mechanism of TID effects on a MOSFET is introduced in Section II. Test structures and the measurement protocol are described in Section III. Then in Section IV, we discuss TID and annealing effects on key device parameters, including the threshold voltage, the free carrier mobility, the subthreshold swing, and the drain-induced barrier lowering (DIBL). The conclusion comes in Section V.

\section{BASIC Physical Processes of TID EFFECTS ON A MOSFET}

When a MOSFET is exposed to high-energy ionizing radiation, electron-hole pairs are produced by the absorbed energy in oxides [16], [17]. Depending on the magnitude of the electric field and the energy of the incident radiation, some of electron-hole pairs undergo recombination immediately. The remaining electrons are swept out of the oxides toward the corresponding electrodes within picoseconds or so. Through a hopping transport mechanism, the remaining holes move through the localized shallow trap sites arising from the disorder of the oxides [18]. This distorts the local potential field of the oxide lattice and tends to confine a fraction of the transporting holes into the relatively deep hole traps (e.g., oxygen vacancies and oxygen interstitials) within the oxide bulk. Oxide trapped charges $\left(\mathrm{Q}_{o t}\right)$, labeled by "+" in Fig. 1, are positive for both $n$ and $p$ MOSFETs. In addition, the holes remaining in the oxide volume form protons $\left(\mathrm{H}^{+}\right)$ via reacting with hydrogen-contained oxide defects [19]. The protons moving to the Si/oxide interfaces break the hydrogen-passivated dangling bonds. This generates electrically active and amphoteric interface defects. Depending on the midgap energy level, interface charged traps $\left(\mathrm{Q}_{i t}\right)$, labeled by " $\times$ " in Fig. 1, are negative in $n$ MOSFETs and positive in $p$ MOSFETs [20].

Fig. 1(b) is the cross section along the length of a MOSFET and illustrates the charge trapping related to gate oxide and spacers. Fig. 1(c) shows the cross section along the width of the MOSFET and presents the charge trapping related to the
TABLE I

DUT LIST

\begin{tabular}{|l|l|l|l|l|l|}
\hline Dimension & DUT1 & DUT2 & DUT3 & DUT4 & DUT5 \\
\hline Width (W) & $3 \mu \mathrm{m}$ & $1 \mu \mathrm{m}$ & $1 \mu \mathrm{m}$ & $1 \mu \mathrm{m}$ & $300 \mathrm{~nm}$ \\
Length (L) & $30 \mathrm{~nm}$ & $30 \mathrm{~nm}$ & $60 \mathrm{~nm}$ & $90 \mathrm{~nm}$ & $30 \mathrm{~nm}$ \\
$\boldsymbol{W} / \boldsymbol{L}$ & 100 & 33.3 & 16.7 & 11.1 & 10 \\
\hline Dimension & DUT6 & DUT7 & DUT8 & DUT9 & DUT10 \\
\hline Width (W) & $100 \mathrm{~nm}$ & $3 \mu \mathrm{m}$ & $400 \mathrm{~nm}$ & $200 \mathrm{~nm}$ & $100 \mathrm{~nm}$ \\
Length (L) & $30 \mathrm{~nm}$ & $1 \mu \mathrm{m}$ & $1 \mu \mathrm{m}$ & $1 \mu \mathrm{m}$ & $1 \mu \mathrm{m}$ \\
$\boldsymbol{W} / \boldsymbol{L}$ & 3.3 & 3 & 0.4 & 0.2 & 0.1 \\
\hline
\end{tabular}

(a)

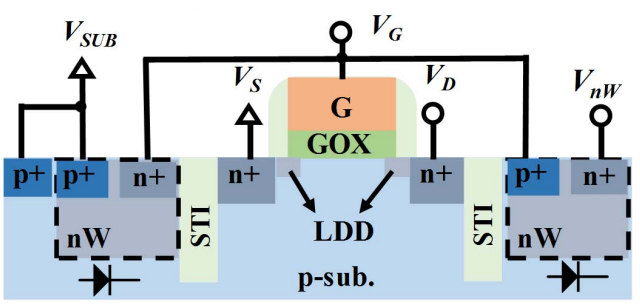

(b)

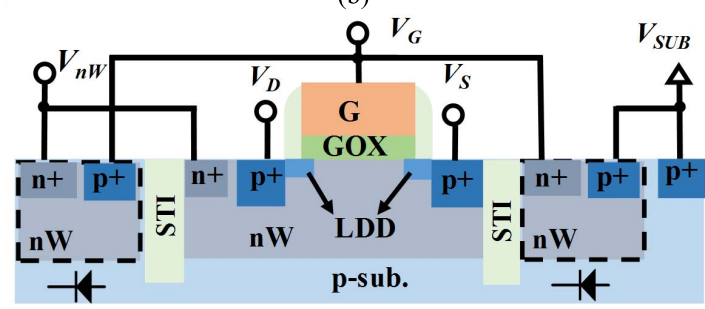

Fig. 2. Cross sections of (a) $n$ and (b) $p$ MOSFETs with two diodes for each as the ESD protection at the gate terminal. The regions with dashed lines form the ESD protection diodes. (a) $n \mathrm{MOS}$ with ESD protection diodes at gate. (b) $p$ MOS with ESD protection diodes at gate.

STI. The charge trapping from both the gate-related dielectrics and the thick isolation oxide influences the device behaviors. In particular, TID effects induce several types of performance degradations, including a threshold voltage shift, a free carrier mobility reduction, a subthreshold swing degradation, and a radiation-modified DIBL.

\section{EXPERIMENTAL DETAILS}

\section{A. Test Structures}

Test chips were fabricated with a commercial 28-nm bulk CMOS process [14], [15]. This paper studies $n$ and $p$ types of standard $V_{T}$ MOSFETs separately with two chips. Each chip has 10 devices under test (DUTs) of the same type, which feature widths ranging from $3 \mu \mathrm{m}$ to $100 \mathrm{~nm}$ and lengths from $1 \mu \mathrm{m}$ to $30 \mathrm{~nm}$, as listed in Table I. Each DUT has one sample that was tested comprehensively. Each MOSFET has two diodes connected to the gate terminal as the electrostatic discharge (ESD) protection, as shown in Fig. 2. Field-oxide FETs are often used for investigating TID effects on the thick STI oxide. Enclosed-layout transistors are often applied for isolating the effects on the STI from the gaterelated dielectrics. However, the strict design rules of this commercial 28-nm bulk CMOS process exclude such special structures. 


\section{B. Measurement Protocol}

The irradiation was conducted at CERN's in-house 10-keV X-ray irradiation system (Seifert RP149). It has a customized probe card with two columns of 16 probe tips installed at a semiautomatic 8-in wafer prober station (Karl-Suss PA200). Through a switching matrix (Keithley 707), two voltage supplies provided the bias for all DUTs during irradiation and annealing. The terminals labeled in Fig. 2 were biased with $V_{G}=V_{D}=V_{n W}=1.1 \mathrm{~V}$ and $V_{S}=V_{S U B}=0$ for $n$ MOSFETs and $V_{G}=V_{D}=V_{S U B}=0$ and $V_{S}=V_{n W}=1.1 \mathrm{~V}$ for $p$ MOSFETs. The temperature-controlled chuck equipped with the irradiation system allows a temperature ranging from $-50{ }^{\circ} \mathrm{C}$ to $200{ }^{\circ} \mathrm{C}$. At a dose rate of $8.82 \mathrm{Mrad} / \mathrm{h}\left(\mathrm{SiO}_{2}\right)$, the chips were irradiated at room temperature $\left(25^{\circ} \mathrm{C}\right)$ up to $1 \mathrm{Grad}$ with steps of $0,10,50,140,340,540,740,940$, 963, and 1000 Mrad. To study the postirradiation annealing effects, the irradiated $n$ MOSFETs were kept at the same temperature $\left(25^{\circ} \mathrm{C}\right)$ with the same bias condition for 450 hours. To speed up the annealing process, $p$ MOSFETs were biased at an elevated temperature $\left(100{ }^{\circ} \mathrm{C}\right)$ for 16 hours. It should be mentioned that with our bias conditions, devices were conducting a high drain current during irradiation. To differentiate the radiation damage from the stress effects, we carried out additional stress tests at the same bias conditions for a comparable time but without irradiation.

Static electrical measurements were performed with the semiconductor parameter analyzer HP4145/55 through the switching matrix at room temperature during irradiation and at the annealing temperature after irradiation. A large number of measurements were conducted to investigate TID effects on all regions of operation. Transfer characteristics were measured from linear $\left(\left|V_{D S}\right|=0.01 \mathrm{~V}\right)$ to saturation $\left(\left|V_{D S}\right|=1.1 \mathrm{~V}\right)$ by sweeping the gate voltage $\left(V_{G}\right)$ from -0.2 to $1.1 \mathrm{~V}$. The output characteristics were measured from depletion region $\left(\left|V_{G S}\right|=0.1 \mathrm{~V}\right)$ to strong inversion $\left(\left|V_{G S}\right|=1.1 \mathrm{~V}\right)$ by sweeping the drain voltage $\left(V_{D}\right)$ from 0 to $1.1 \mathrm{~V}$. Since the radiation damage anneals with time [21], a voltage step of $25 \mathrm{mV}$ was chosen as a suitable compromise between limiting the measurement duration and providing a sufficient measurement resolution. In this paper, the on-current, the threshold voltage, the free carrier mobility, the off-current, and the subthreshold swing are extracted from the measured drain current $\left(I_{D}\right)$ versus $V_{G}$ curves in the saturation operation. The DIBL parameter is obtained from the $I_{D}-V_{G}$ curves with 0.01 and $1.1 \mathrm{~V}$ of $\left|V_{D S}\right| . p \mathrm{DUT} 10$ demonstrates an unexpected abrupt increase in the off-current around $140 \mathrm{Mrad}$ that is not seen for other DUTs. Due to the fact that its source and substrate are connected with other DUTs and its gate is coupled with the ESD protection diodes, we are not able to obtain the intrinsic currents from gate, source, and substrate. Therefore, it is not straightforward to clearly identify the source of this off-current increase. In order not to mislead the reader by the suspect behavior of $p$ DUT10, we exclude it from our discussion. Note that $n$ MOSFETs lost the bias for hours between 540 and 740 Mrad. pMOSFETs were not biased for hours between 940 and $963 \mathrm{Mrad}$. This bias loss explains the discontinuous evolution of the extracted parameters.

\section{RESUlTS AND Discussion}

\section{A. Bias Dependence of TID Effects}

TID effects strongly depend on the applied electric field. It is reported in [9] that for a commercial 65-nm bulk CMOS process, the real worst bias cases are $V_{G B}=V_{D S}=V_{D D}$ for $n$ MOSFETs and $V_{G B}=V_{D S}=-V_{D D}$ for $p$ MOSFETs that are different from the historically worst bias cases $\left(V_{G B}=1.1 \mathrm{~V}, V_{D S}=0\right.$ for $n$ MOSFETs and $V_{G B}=V_{D S}=0$ for $p$ MOSFETs [22]). We compare the effects of these two bias conditions on 28-nm bulk $n$ and pMOSFETs. No big difference is seen between them and the slight difference is actually within the chip variability $(\sim 6 \%)$. It is not straightforward to decide which are the worst bias cases for this 28-nm bulk CMOS process. However, in most analog circuits and particularly the analog FE electronics used in HEP, the transistors are biased in saturation with a nonzero $V_{D S}$ except the switches working with a zero $V_{D S}$. In order to reproduce as close as possible the realistic bias conditions for analog circuits, we therefore chose to bias the devices with $V_{G B}=V_{D S}=1.1 \mathrm{~V}$ for $n$ MOSFETs and $V_{G B}=V_{D S}=-1.1 \mathrm{~V}$ for $p$ MOSFETs.

Since the devices conducted a high drain current during irradiation with our bias conditions, it is of importance to see how much of the performance degradation comes from TID effects. For $n$ MOSFETs, the stress without irradiation does not cause any performance degradation, leaving the whole range of $I_{D}-V_{G}$ curves unaffected. Therefore, the performance degradation of the irradiated $n$ MOSFETs is attributed only to the radiation effects. However, $p$ MOSFETs demonstrate some stress-induced shift in the threshold voltage. The ratio of the stress effects to the radiation damage depends on the transistor dimension. However, except the largest pMOSFETs $(3 \mu \mathrm{m} / 1 \mu \mathrm{m})$ for which the damage is mostly due to the stress, the degradation of all the others is dominated by TID effects $(70 \%-95 \%)$. Additionally, there might be selfheating during irradiation due to the high conducting current. However, between the end of the irradiation and the start of the first DC measurement, the time (a couple of seconds) should be sufficient for the chips to cool down to the room temperature [23]. Since the self-heating normally appears at the high current level, we believe that the important parameters extracted from the low current region of the measured $I_{D^{-}} V_{G}$ curves, such as the threshold voltage, the free carrier mobility, the subthreshold swing, and the off-current, are not affected by self-heating. Nevertheless, we are mostly interested in the realistic bias conditions that are close to the real circuit operating regime, even though there might be self-heating affecting the on-current.

Section III-B mentioned the bias loss between 540 and 740 Mrad for $n$ MOSFETs and 940 and 963 Mrad for $p$ MOSFETs that causes the parameter discontinuities. Electron tunneling from the oxides to the substrate is expected to be the underlying mechanism. At a positive gate-to-bulk bias, electrons tunnel from the substrate to electron traps in oxides and neutralize the associated holes that are mostly trapped at near-interfacial oxide traps (i.e., border traps). Removing the bias, these electrons tunnel back to the substrate and lead to 


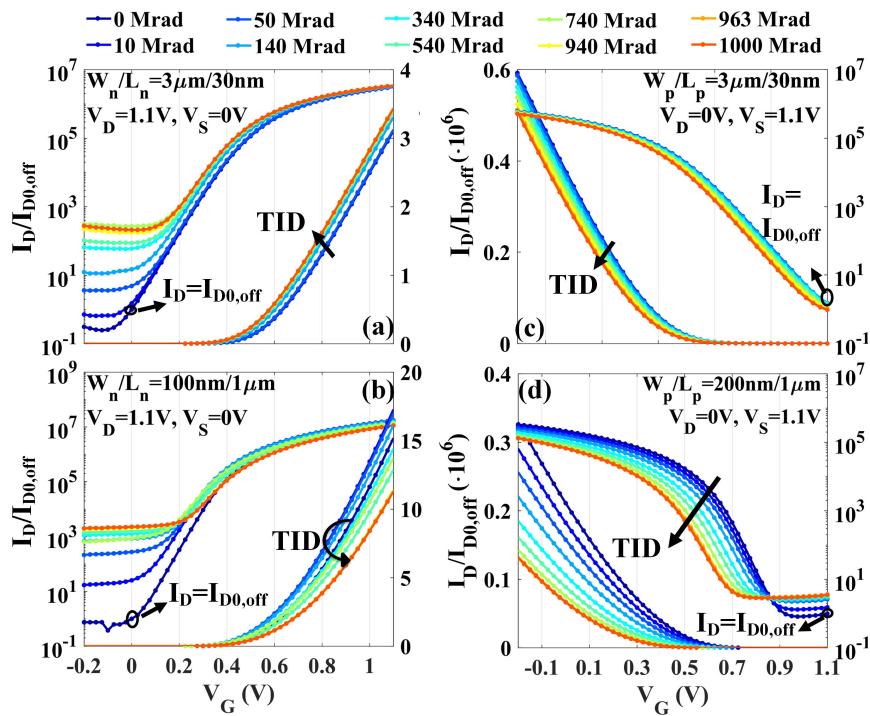

Fig. 3. Transfer characteristics of $n$ (a), (b) and $p$ (c), (d) types of MOSFETs in saturation mode $\left(\left|V_{D S}\right|=1.1 \mathrm{~V}\right)$ with respect to TID (a) and (c) are for DUT1 with the largest $W / L$ ratio $(3 \mu \mathrm{m} / 30 \mathrm{~nm})$. (b) is for $n$ DUT10 with the smallest $W / L$ ratio $(100 \mathrm{~nm} / 1 \mu \mathrm{m})$ and (d) is for $p$ DUT9 with $100 \mathrm{~nm} / 1 \mu \mathrm{m}$. $I_{D 0, \text { off } f}$ is the off-current $\left(V_{G S}=0\right)$ before irradiation.

a higher amount of oxide trapped charges. This decreases the threshold voltage, increases the on-current, and degrades the subthreshold swing. The negative threshold voltage shift also leads to a higher off-current for short-channel $n$ MOSFETs. Long-channel $n$ MOSFETs present a lower off-current, which could be explained by the compensation of the interface charge trapping. The bias loss between 940 and $963 \mathrm{Mrad}$ for $p$ MOSFETs explains the slight fluctuation of the extracted parameters for the last two TID steps.

\section{B. TID Effects on Transfer Characteristics}

Fig. 3 plots the normalized drain current of $n$ DUT1, $n$ DUT10, $p$ DUT1, and $p$ DUT9 with respect to TID. The on-current $\left(I_{D, \text { on }}\right)$ is defined at $\left|V_{G S}\right|=1.1 \mathrm{~V}$, and the off-current $\left(I_{D, o f f}\right)$ at $V_{G S}=0$. $n \mathrm{DUT} 1$ has a negative threshold voltage shift and a monotonic on-current increase, as shown in Fig. 3(a). Nevertheless, as seen in Fig. 3(b), the threshold voltage of $n$ DUT10 first decreases and then increases, leading to the corresponding on-current evolution. Both irradiated $n$ DUT1 and $n$ DUT10 have a significant off-current increase. In contrast, the threshold voltage of both $p$ DUT1 [Fig. 3(c)] and $p$ DUT9 [Fig. 3(d)] decreases with TID, leading to a continuous on-current loss. Note that when the threshold voltage of a $p$ MOSFET decreases, it becomes more negative and its absolute value increases. The off-current of both $p$ DUT1 and $p$ DUT9 is not sensitive to TID and has a slight off-current change.

\section{On-current}

The relative on-current variation is plotted as a function of TID in Fig. 4. All $n$ MOSFETs present an on-current improvement at a lower TID ( 10 Mrad) in Fig. 4(a). Then

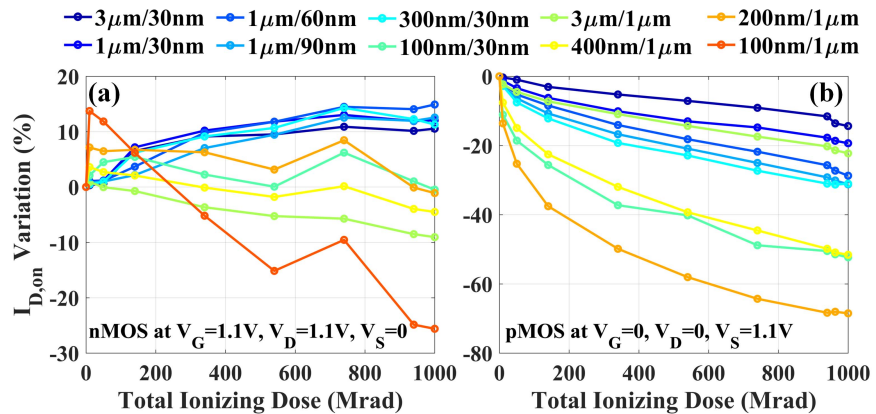

Fig. 4. TID-induced on-current $\left(I_{D, o n}\right)$ variation of (a) $n$ and (b) $p$ types of MOSFETs in saturation mode $\left(\left|V_{D S}\right|=1.1 \mathrm{~V}\right)$. $I_{D \text {, on }}$ is obtained at $\left|V_{G S}\right|=1.1 \mathrm{~V}$.

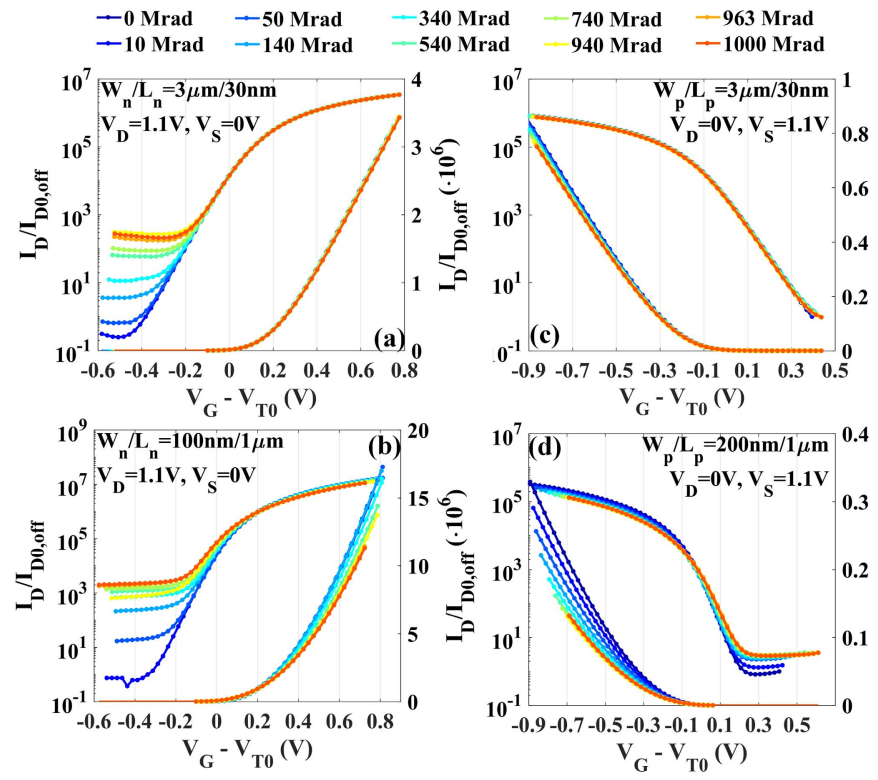

Fig. 5. Drain current $\left(I_{D}\right)$ versus the overdrive voltage $\left(V_{G}-V_{T 0}\right)$ with respect to TID for (a) and (b) $n$ and (c) and (d) $p$ types of MOSFETs in saturation mode $\left(\left|V_{D S}\right|=1.1 \mathrm{~V}\right)$.

for $n$ DUT1-5, the on-current increase continues until reaching a $\sim 15 \%$ increase. However, the on-current of $n$ DUT6-10 starts to decrease at a higher TID. $n$ DUT10 with the smallest $W / L$ ratio $(100 \mathrm{~nm} / 1 \mu \mathrm{m})$ evidences as the most dynamic case with a maximum on-current loss of $\sim 25 \%$. Fig. 4(b) shows that $p$ MOSFETs are grouped into two distinct categories: $p$ DUT1-6 and $p$ DUT7-9. In each group, the narrower the channel, the higher the on-current loss. Eventually, most of $p$ MOSFETs show a less than $30 \%$ degradation in the on-current. Narrowchannel $p$ MOSFETs with a smaller $W / L$ ( $p$ DUT6 and $p$ DUT8-9) present a more than 50\% on-current loss. Nevertheless, these results are much better compared with the commercial $65-\mathrm{nm}$ bulk CMOS technology from the same foundry [9].

Both the threshold voltage $\left(V_{T 0}\right)$ shift and the free carrier mobility $(\mu)$ reduction influence the on-current. To extract $V_{T 0}$ and $\mu, \sqrt{I_{D}}-V_{G}$ curves are extrapolated linearly at the maximum slope. The intercept at the $V_{G}$ axis is defined as $V_{T O}$. The slope of the linear extrapolation provides insights into $\mu$. 


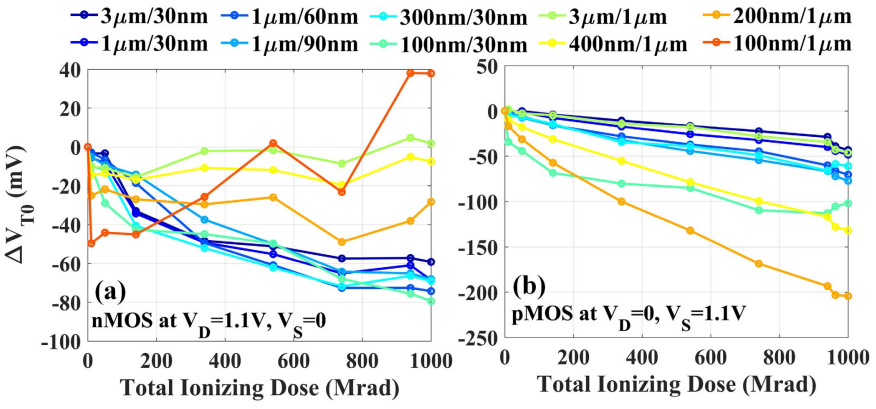

Fig. 6. TID-induced threshold voltage ( $\left.V_{T 0}\right)$ shift of (a) $n$ and (b) $p$ types of MOSFETs in saturation mode $\left(\left|V_{D S}\right|=1.1 \mathrm{~V}\right)$.

We now plot $I_{D}$ with respect to $V_{G}-V_{T 0}$ in Fig. 5 to isolate the effects of the free carrier mobility reduction from the threshold voltage shift. It is seen in Fig. 5(a) and (c) that for the widest/shortest channel DUTs $(3 \mu \mathrm{m} / 30 \mathrm{~nm})$, the $I_{D}$ versus $V_{G}-V_{T 0}$ curves overlap in strong inversion, demonstrating that only the threshold voltage shift accounts for the on-current variation. For the narrowest/longest channel DUTs, as shown in Fig. 5(b) and (d), the $I_{D}$ versus $V_{G}-V_{T 0}$ curves deviate in strong inversion, indicating the additional contribution of the mobility reduction to the on-current loss. We also suspect that for short-channel $p$ MOSFETs, the positive charges trapped in the spacers reduce the effective doping of the lightly-doped drain region and increase the resistance for the drain current. This might partly contribute to the significant on-current loss of $p$ DUT6 $(100 \mathrm{~nm} / 30 \mathrm{~nm})$. However, with our current measurements, we are not able to isolate the effects of the spacers from the gate oxide. Further investigations are therefore needed to verify if this technology does undergo the effects of the charge buildup in the spacers.

1) Threshold Voltage: $\mathrm{Q}_{o t}$ decreases the threshold voltage of both $n$ and $p$ MOSFETs, whereas $\mathrm{Q}_{i t}$ increases that of $n$ MOSFETs and decreases that of $p$ MOSFETs. For $n$ MOSFETs, the counterbalance of $\mathrm{Q}_{o t}$ and $\mathrm{Q}_{i t}$ leads to a limited influence on the switched-on region. Fig. 6(a) shows a less than $80-\mathrm{mV}$ shift in the threshold voltage, corresponding to a less than $30 \%$ variation. This explains the limited on-current variation in Fig. 4(a). It is also observed that at a lower TID, the threshold voltage decreases for all $n$ MOSFETs, which is due to the initial oxide trapped charges. Then the threshold voltage of $n$ DUT1- 6 continues to decrease until 1 Grad. For $n$ DUT7-10, $\mathrm{Q}_{i t}$ starts at a certain TID to compensate for the $\mathrm{Q}_{o t}$-induced negative threshold voltage shift. Eventually, $n$ DUT10 has an overall positive threshold voltage shift that is consistent with the net on-current decrease. Fig. 6(b) shows that even with the superposed effects of $\mathrm{Q}_{o t}$ and $\mathrm{Q}_{i t}$, most of $p$ MOSFETs still have a threshold voltage shift of less than $80 \mathrm{mV}$. However, the threshold voltage of narrow-channel $p$ MOSFETs with a smaller $W / L$ ratio ( $p$ DUT6 and $p$ DUT8-9) decreases by more than $100 \mathrm{mV}$ that corresponds to their significant on-current loss. It should be mentioned that the threshold voltage variation for all $p$ MOSFETs is within $30 \%$. The significant effect on narrow-channel $p$ MOSFETs is attributed to the charge trapping

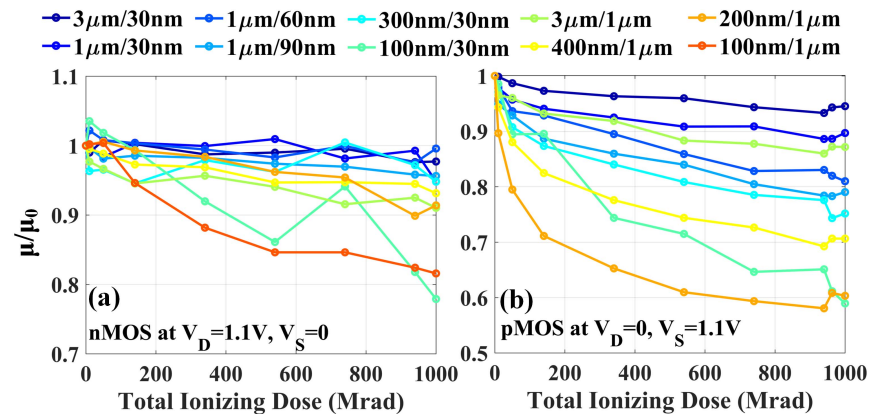

Fig. 7. TID-induced free carrier mobility $(\mu)$ reduction of (a) $n$ and (b) $p$ types of MOSFETs in saturation mode $\left(\left|V_{D S}\right|=1.1 \mathrm{~V}\right) . \mu_{0}$ is the free carrier mobility before irradiation.

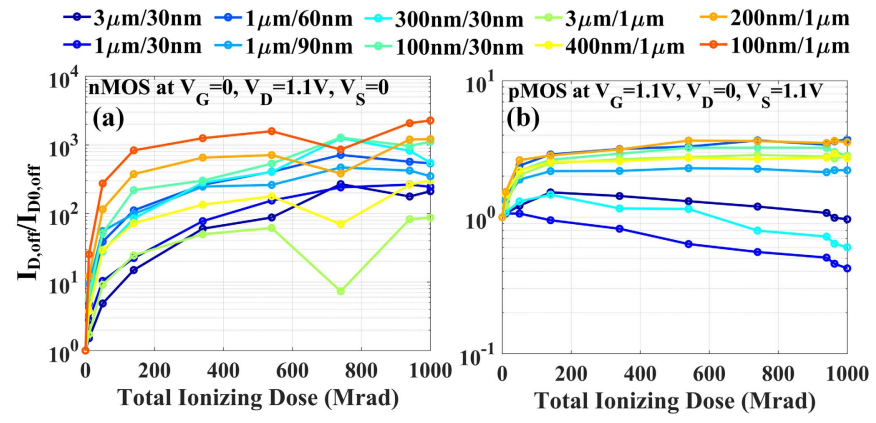

Fig. 8. TID-induced off-current $\left(I_{D, \text { off }}\right)$ increase of (a) $n$ and (b) $p$ types of MOSFETs in saturation mode $\left(\left|V_{D S}\right|=1.1 \mathrm{~V}\right) . I_{D \text {,off }}$ is obtained at $V_{G S}=0$.

in the thick STI oxide, which modifies the electric field at the STI edges and prevents the inversion of the silicon channel [9], [11], [24].

2) Free Carrier Mobility: The radiation-induced mobility reduction demonstrates a strong dependence on the Coulomb scattering of the trapped charges at border traps [25] and interface traps [26]. Fig. 7(a) shows that wide-/short-channel MOSFETs have a negligible mobility reduction, which indicates the radiation hardness of the interface of the gate oxide and the silicon channel. The significant negative threshold voltage shift in Fig. 6(a) actually indicates a large amount of trapped charges at border traps in $n$ DUT6 and at interface traps in $n$ DUT10, which contributes to their high mobility reduction. Compared with $n$ MOSFETs, $p$ MOSFETs demonstrate a higher mobility reduction, as seen in Fig. 7(b). The mobility of $p$ DUT6 and $p$ DUT8-9 degrades more that is consistent with their significant on-current loss depicted in Fig. 4(b). The highest mobility reduction in narrowest/ longest channel DUTs ( $n$ DUT10 and $p$ DUT9) is clearly illustrated by the downbending of the $I_{D}-\left(V_{G}-V_{T 0}\right)$ curves in Fig. 5(b) and (d).

\section{Off-current}

The off-current normalized to preirradiation values is plotted as a function of TID in Fig. 8. Fig. 8(a) demonstrates a significant off-current increase by two to three orders of magnitude for $n$ MOSFETs. It should be mentioned that different mechanisms are dominating the region around a zero $V_{G S}$ for shortand long-channel $n$ MOSFETs, as seen in Fig. 3(a) and (b). 


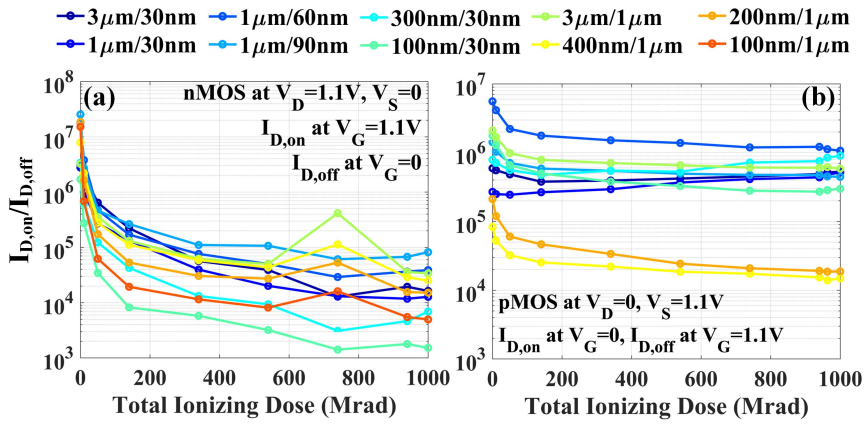

Fig. 9. TID-induced on/off-current ratio $\left(I_{D, \text { on }} / I_{D, \text { off }}\right)$ degradation of (a) $n$ and (b) $p$ types of MOSFETs in saturation mode $\left(\left|V_{D S}\right|=1.1 \mathrm{~V}\right)$.

The extracted off-current of short-channel $n$ MOSFETs is still in the subthreshold region before irradiation. At a lower TID, trapped holes in the thick STI oxide deplete the channel along the STI edges. This connects the source/drain depletion regions and results in a surface punchthrough current [27]. At a higher TID, the positive charges trapped in the thick STI oxide enhance the DIBL and further increase the off-current. The radiation-enhanced DIBL will be discussed in detail in Section IV-F.

For long-channel $n$ MOSFETs, the dominant off-current contributor is the parasitic leakage current along the STI edges [11]. Trapped holes in the thick STI oxide could be strong enough to invert the adjacent $p$-type substrate and to form the $n$-channel. Thus, two parasitic leakage paths form even when the main transistor is switched off. This effect has a stronger influence on a narrow channel, as seen with the highest off-current increase for $n$ DUT10. Furthermore, up to a certain TID, the off-current of long-channel $n$ MOSFETs enters into a region less dependent on the gate voltage. It almost saturates up to $340 \mathrm{Mrad}$ that indicates the complete filling of the traps in the STI oxide or the compensation of the interface charge trapping from the STI. It should also be emphasized that it is more difficult to invert a highly doped silicon. Therefore, the nonuniform doping profile in nanoscale MOSFETs, including the increased channel doping concentration due to the scaling rule, the surface implantation for adjusting the threshold voltage, and the higher doping from the retrograde well and the halo pockets for suppressing short-channel effects, could be advantageous in terms of the radiation tolerance at the switched-off state [28], [29]. For all $n$ MOSFETs, the radiation-induced charge buildup is not strong enough to influence the electrostatic potential in the middle of the channel. Therefore, as shown in Fig. 9(a), there is still an on/off-current ratio of three orders of magnitude, leaving a sufficient margin for circuit design.

Compared with $n$ MOSFETs, the switched-off region of $p$ MOSFETs is much less susceptible to TID. The off-current changes only by a maximum factor of three. The slight off-current increase might be from the peripheral drain to substrate junction leakage current. It is mostly contributed by the surface generation at the intersection of the depletion region and the STI sidewalls where a high density of interface traps are located [30]. Some of the short-channel

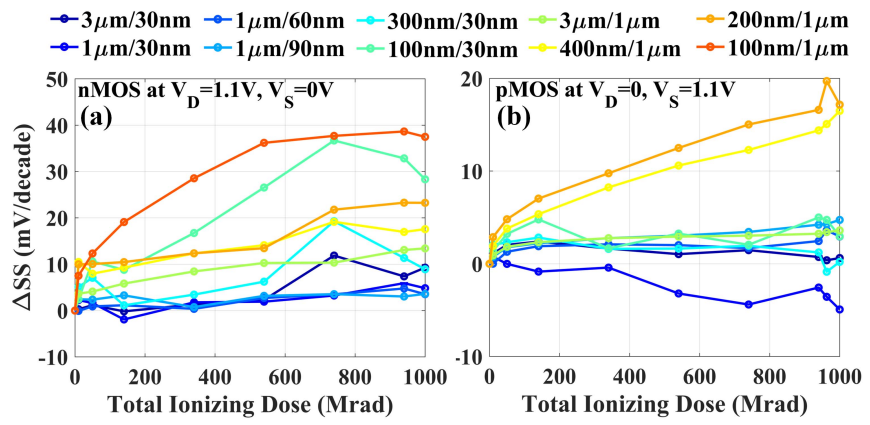

Fig. 10. TID-induced subthreshold swing (SS) degradation of (a) $n$ and (b) $p$ types of MOSFETs in saturation mode $\left(\left|V_{D S}\right|=1.1 \mathrm{~V}\right)$.

$p$ MOSFETs present a slight off-current decrease. This is due to the fact that short-channel $p$ MOSFETs still work in the subthreshold region around a zero $V_{G S}$, as shown in Fig. 3(c) for $p$ DUT1. The negative threshold voltage shift together with the radiation-suppressed DIBL (Section IV-F) leads to the off-current decrease. Owing to the negligible influence on the off-current, the irradiated $p$ MOSFETs still demonstrate an on/off-current ratio of more than four orders of magnitude, as shown in Fig. 9(b).

\section{E. Subthreshold Swing}

Trapped charges at border traps and interface traps cut off part of the electric field lines from the gate electrode. This reduces the effective gate potential and the transistors could not be switched off effectively. Fig. 10 shows a less than $20 \mathrm{mV} /$ decade increase in the subthreshold swing for most of $n$ and $p$ MOSFETs. This confirms the radiation hardness of the interface of the gate oxide and the silicon channel. Two narrowest-channel $n$ MOSFETs ( $n$ DUT6 and $n$ DUT10) demonstrate a $\sim 40 \mathrm{mV} /$ decade subthreshold swing increase. Note that the higher increase in the off-current of $n$ DUT6 and $n$ DUT10 raises up the $I_{D}-V_{G}$ curves and starts to mask the subthreshold region. This degrades the precision of the subthreshold swing extraction.

\section{F. Drain-Induced Barrier Lowering}

The DIBL represents one of the most fundamental short-channel effects in nanoscale MOSFETs. The STI-related charge trapping is among the most important DIBL factors [27]. In the proposed dipole theory [31], $\mathrm{Q}_{o t}$ in the thick STI oxide terminates the drain-to-gate fringing field at the switched-off state. This decreased drain-to-gate coupling in turn enhances the drain-to-source coupling. In addition, $\mathrm{Q}_{o t}$ in the thick STI oxide enhances the electric field along the STI edges. Thus, the electric field lines in the center of the channel will be denser and the drain-to-source coupling will be further strengthened. Both enhance the DIBL of $n$ MOSFETs, as shown in Fig. 11(a).

As mentioned in Section IV-D, the STI-related charge trapping makes the parasitic depletion regions interact with the source/drain ones and forms a thicker depletion region in short-channel $n$ MOSFETs. This will shorten the effective channel length near the corners of the STI and the gate oxide, 


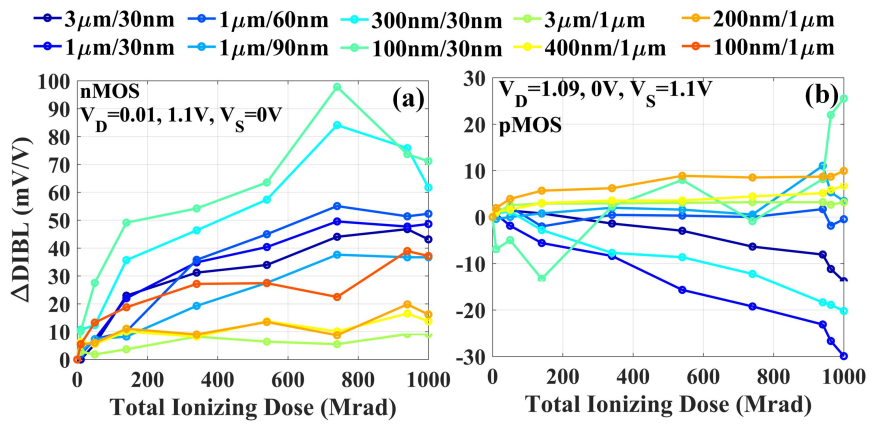

Fig. 11. TID-modified DIBL of (a) $n$ and (b) $p$ types of MOSFETs. The DIBL is extracted as DIBL $=-\left(V_{T 0}^{\left|V_{D S}\right|=1.1 \mathrm{~V}}-V_{T 0}^{\left|V_{D S}\right|=0.01 \mathrm{~V}}\right) /(1.1-0.01 \mathrm{~V})$.
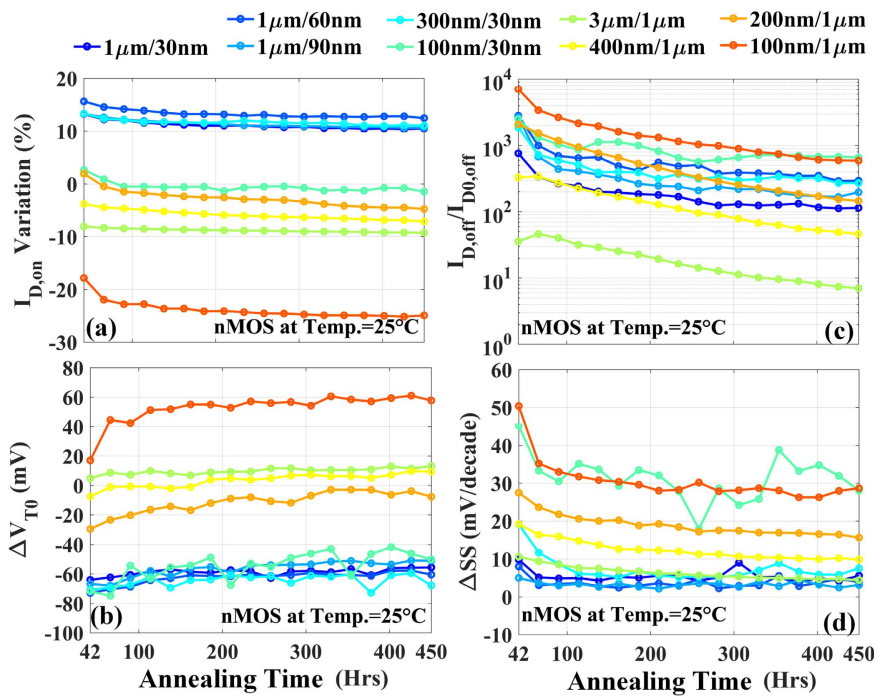

Fig. 12. Postirradiation annealing effects on the on-current $\left(I_{o n}\right)$, the threshold voltage $\left(V_{T 0}\right)$, the off-current $\left(I_{O f f}\right)$, and the subthreshold swing $(S S)$ of $n$ MOSFETs. There is no result for the widest/shortest channel $n$ MOSFET ( $3 \mu \mathrm{m} / 30 \mathrm{~nm}$ ) due to the physical damage from moving and reconnecting the chip.

adding another enhancing factor for the DIBL. If the width is narrow, trapped charges in the thick STI oxide even raise the nearby body potential in the main channel, which significantly lowers down the potential barriers with source and drain. This is shown in Fig. 11(a): the narrower the channel, the more severe the radiation-enhanced DIBL.

The radiation has a negligible influence on the DIBL of most of $p$ MOSFETs, as seen in Fig. 11(b). However, $p$ DUT1-2 and $p$ DUT5 demonstrate a radiation-suppressed DIBL. This could be explained by the proposed dipole theory. Both the trapped charges in the thick STI oxide and the image charges in the gate electrode are positive. This weakens the drain-to-source coupling and suppresses the DIBL [32]. Due to STI-related trapped charges, electrons accumulate around the corners of the gate oxide and the STI. This will lengthen the effective channel near the edges, which further decreases the DIBL of $p$ DUT1-2 and $p$ DUT5.

\section{G. Postirradiation Annealing}

The long-term postirradiation annealing is believed to annihilate or neutralize the oxide trapped charges even at room

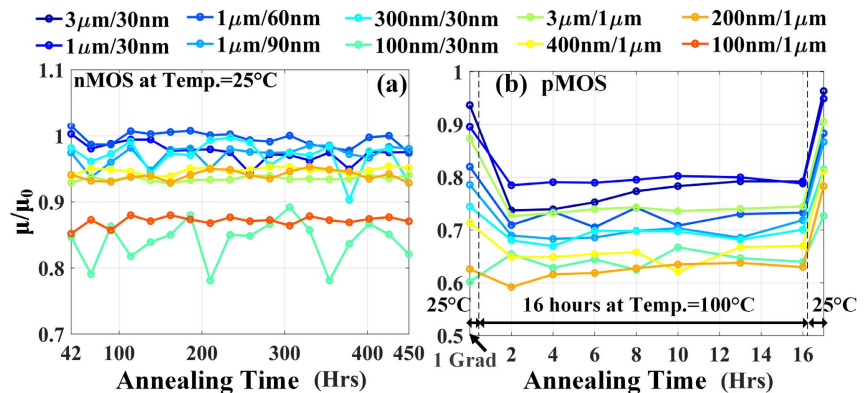

Fig. 13. Postirradiation annealing effects on the free carrier mobility $(\mu)$ of (a) $n$ and (b) $p$ types of MOSFETs. In (b), the first measurement corresponds to $1 \mathrm{Grad}$ at room temperature. The second is with respect to 2 hours of hightemperature annealing. The last is a room temperature measurement after the high-temperature annealing. The dashed lines indicate the changing of the temperature.

temperature [33]. This annealing process can be accelerated by a stronger positive gate-to-bulk bias and a higher temperature [34]. It is reported that in some cases the interface-trap annealing can happen below $100{ }^{\circ} \mathrm{C}$ [35]. However, a higher temperature is generally required to anneal interface traps effectively [36].

Figs. 12 and 13(a) present the room temperature $\left(25^{\circ} \mathrm{C}\right)$ annealing effects on important parameters of $n$ MOSFETs. The first measurement was taken after 42 hours of annealing. Since the room temperature is not high enough to anneal the interface traps, the observation gives more insights into the evolution of the oxide trapped charges. The threshold voltage of $n$ MOSFETs increases due to the trapped-hole annealing, as observed in Fig. 12(b). Fig. 13(a) demonstrates a negligible change in the free carrier mobility, which confirms no annealing evolution for radiation-induced interface traps. Therefore, the room temperature annealing decreases the on-current of $n$ MOSFETs, as seen in Fig. 12(a). The trapped-hole annealing also decreases the off-current significantly, as shown in Fig. 12(c). An observable improvement in the subthreshold swing is seen in Fig. 12(d), indicating the annealing of the trapped holes at border traps. The remaining subthreshold swing degradation for $n$ DUT6 and $n$ DUT810 could be attributed to the interface charged traps at the STI/channel edges.

Figs.14 and 13(b) present the high-temperature $\left(100{ }^{\circ} \mathrm{C}\right)$ annealing effects on important parameters of $p$ MOSFETs. The chip was annealed at $100{ }^{\circ} \mathrm{C}$ for 16 hours and then cooled down to $25{ }^{\circ} \mathrm{C}$. The first measurement point corresponds to $1 \mathrm{Grad}$ at room temperature. The second refers to 2 hours of high-temperature annealing. The big differences between these two sets of measurement points include the effects of the temperature dependence that is demonstrated as a higher off-current, an increased threshold voltage, a degraded subthreshold swing, and a reduced free carrier mobility. The oncurrent increase is due to the dominant effects of the positive threshold voltage shift over the free carrier mobility reduction. Since the subthreshold swing increase is less than 1.25 that is the temperature ratio of the first two measurements, the second measurement point also includes the effect of high-temperature annealing. In the following hours, the degraded performance 


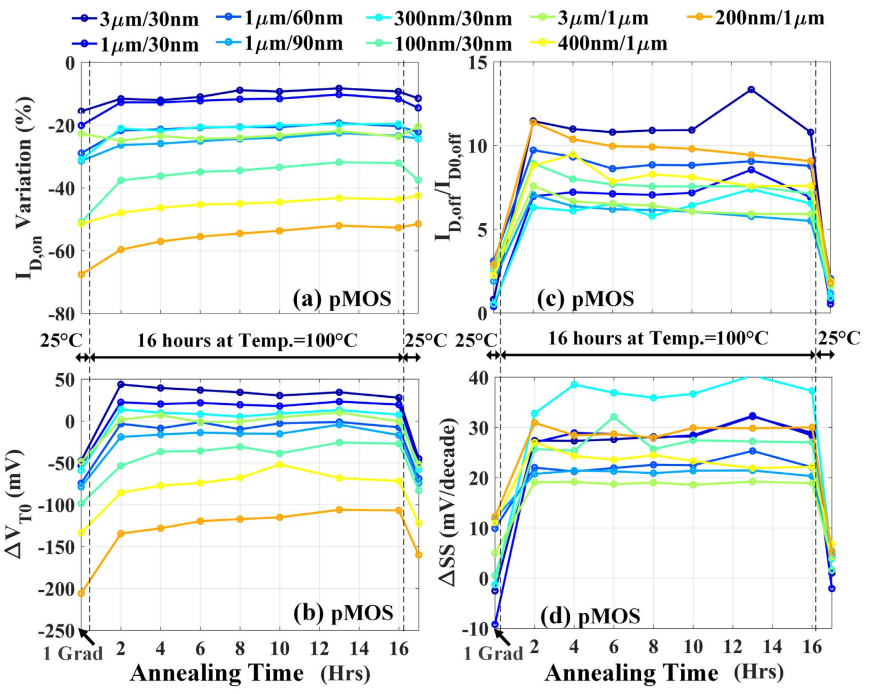

Fig. 14. Postirradiation annealing effects on the on-current $\left(I_{o n}\right)$, the threshold voltage $\left(V_{T 0}\right)$, the off-current $\left(I_{O f f}\right)$, and the subthreshold swing (SS) of $p$ MOSFETs.

of $p$ MOSFETs is partly recovered. The threshold voltage [Fig. 14(b)] increases and the free carrier mobility improves slightly [Fig. 13(b)], which leads to an observable on-current increase [Fig. 14(a)]. The differences between two room temperature measurements demonstrate the net performance improvements. It is observed that after 16 hours of hightemperature annealing, the threshold voltage increases by up to $40 \mathrm{mV}$, the mobility improves by up to $12 \%$, and the on-current recovers by up to $20 \%$. The off-current and the subthreshold swing almost reach the original values.

\section{CONCLUSion}

The effects of TID up to $1 \mathrm{Grad}$ and postirradiation annealing on 28-nm bulk $n$ and $p$ MOSFETs are investigated. The counterbalance of interface and oxide charge trapping results in a limited influence on the switched-on region of the irradiated $n$ MOSFETs, which demonstrate a less than $25 \%$ free carrier mobility reduction, a less than $80-\mathrm{mV}$ threshold voltage shift, and a less than $25 \%$ on-current loss. However, due to the STI-induced parasitic leakage paths and the radiationenhanced DIBL, $n$ MOSFETs present a two to three orders of magnitude increase in the off-current. In contrast, most of $p$ MOSFETs have a negligible off-current change. Even with the superposed effects of interface and oxide charged traps, most of $p$ MOSFETs still undergo a less than $30 \%$ performance degradation, which is much less compared with the $65-\mathrm{nm}$ counterparts. Owing to the radiation-induced narrow channel effects, the narrowest/longest channel $p$ MOSFET presents the strongest susceptibility to TID with a $200-\mathrm{mV}$ threshold voltage shift and a $40 \%$ free carrier mobility reduction as well as a $70 \%$ on-current loss. The limited subthreshold swing degradation indicates the strong radiation tolerance of the interface of the gate dielectric and the silicon channel.

Due to the complex interplay of the charge trapping relevant to the gate-related dielectrics and the STI, TID effects demonstrate a strong geometry dependence. This implies the importance of choosing the suitable sizes of transistors for radiation-tolerant circuit design. In addition, oxide trapped holes anneal with time, which leads to a significant off-current decrease in $n$ MOSFETs and a substantial on-current recovery in $p$ MOSFETs. Considering the long-term irradiation and annealing in realistic applications, the degradation might be mitigated.

\section{ACKNOWLEDGMENT}

The authors would like to thank Dr. A. Marchioro from the CERN EP-ESE Group for the fruitful collaborations.

\section{REFERENCES}

[1] J. Butler et al., "CMS phase II upgrade scope document," CERN, Geneva, Switzerland, Tech. Rep. CERN-LHCC-2015-019, 2015.

[2] K. Einsweiler and L. Pontecorvo, "ATLAS phase-II upgrade scoping document," CERN, Geneva, Switzerland, Tech. Rep. CERN-LHCC2015-020, Sep. 2015.

[3] F. Ellinger, M. Claus, M. Schröter, and C. Carta, "Review of advanced and beyond CMOS FET technologies for radio frequency circuit design," in Proc. SBMO/IEEE MTT-S Int. Microw. Optoelectron. Conf. (IMOC), Oct. 2011, pp. 347-351.

[4] J. M. Benedetto, H. E. Boesch, F. B. McLean, and J. P. Mize, "Hole removal in thin-gate MOSFETs by tunneling," IEEE Trans. Nucl. Sci., vol. NS-32, no. 6, pp. 3916-3920, Dec. 1985.

[5] K. Choi et al., "The past, present and future of high- $k$ /metal gates," ECS Trans., vol. 53, no. 3, pp. 17-26, 2013.

[6] A. Y. Kang, P. M. Lenahan, and J. F. Conley, "The radiation response of the high dielectric-constant hafnium oxide/silicon system," IEEE Trans. Nucl. Sci., vol. 49, no. 6, pp. 2636-2642, Dec. 2002.

[7] S. K. Dixit et al., "Radiation induced charge trapping in ultrathin $\mathrm{HfO}_{2}$-based MOSFETs," IEEE Trans. Nucl. Sci., vol. 54, no. 6, pp. 1883-1890, Dec. 2007.

[8] G. X. Duan et al., "Bias dependence of total ionizing dose effects in SiGe-SiO $2 / \mathrm{HfO}_{2}$ pMOS FinFETs," IEEE Trans. Nucl. Sci., vol. 61, no. 6, pp. 2834-2838, Dec. 2014.

[9] F. Faccio, S. Michelis, D. Cornale, A. Paccagnella, and S. Gerardin, "Radiation-induced short channel (RISCE) and narrow channel (RINCE) effects in 65 and $130 \mathrm{~nm}$ MOSFETs," IEEE Trans. Nucl. Sci., vol. 62, no. 6, pp. 2933-2940, Dec. 2015.

[10] M. Menouni et al., "1-Grad total dose evaluation of $65 \mathrm{~nm}$ CMOS technology for the HL-LHC upgrades," J. Instrum., vol. 10, no. 5, p. C05009, 2015.

[11] F. Faccio and G. Cervelli, "Radiation-induced edge effects in deep submicron CMOS transistors," IEEE Trans. Nucl. Sci., vol. 52, no. 6, pp. 2413-2420, Dec. 2005.

[12] M. Gaillardin, V. Goiffon, S. Girard, M. Martinez, P. Magnan, and P. Paillet, "Enhanced radiation-induced narrow channel effects in commercial $0.18 \mu \mathrm{m}$ bulk technology," IEEE Trans. Nucl. Sci., vol. 58, no. 6, pp. 2807-2815, Dec. 2011.

[13] M. Gaillardin et al., "Investigations on the vulnerability of advanced CMOS technologies to MGy dose environments," IEEE Trans. Nucl. Sci., vol. 60, no. 4, pp. 2590-2597, Aug. 2013.

[14] A. Pezzotta et al., "Impact of GigaRad ionizing dose on $28 \mathrm{~nm}$ bulk MOSFETs for future HL-LHC," in Proc. 46th Eur. Solid-State Device Res. Conf. (ESSDERC), Sep. 2016, pp. 146-149.

[15] C.-M. Zhang et al., "GigaRad total ionizing dose and post-irradiation effects on $28 \mathrm{~nm}$ bulk MOSFETs," in Proc. IEEE Nucl. Sci. Symp. Conf. Rec., Nov. 2016, pp. N43-N48.

[16] D. M. Fleetwood, "Total ionizing dose effects in MOS and low-doserate-sensitive linear-bipolar devices," IEEE Trans. Nucl. Sci., vol. 60 , no. 3, pp. 1706-1730, Jun. 2013.

[17] J. R. Schwank et al., "Radiation effects in MOS oxides," IEEE Trans. Nucl. Sci., vol. 55, no. 4, pp. 1833-1853, Aug. 2008.

[18] R. C. Hughes, "Time-resolved hole transport in a-SiO 2 ," Phys. Rev. B, Condens. Matter, vol. 15, no. 4, p. 2012, 1977.

[19] N. S. Saks, R. B. Klein, and D. L. Griscom, "Formation of interface traps in MOSFETs during annealing following low temperature irradiation," IEEE Trans. Nucl. Sci., vol. NS-35, no. 6, pp. 1234-1240, Dec. 1988.

[20] P. J. McWhorter and P. S. Winokur, "Simple technique for separating the effects of interface traps and trapped-oxide charge in metal-oxidesemiconductor transistors," Appl. Phys. Lett., vol. 48, no. 2, pp. 133-135, 1986. 
[21] G. F. Derbenwick and H. H. Sander, "CMOS hardness prediction for low-dose-rate environments," IEEE Trans. Nucl. Sci., vol. NS-24, no. 6, pp. 2244-2247, Dec. 1977.

[22] P. V. Dressendorfer, J. M. Soden, J. J. Harrington, and T. V. Nordstrom, "The effects of test conditions on MOS radiation-hardness results," IEEE Trans. Nucl. Sci., vol. NS-28, no. 6, pp. 4281-4287, Dec. 1981.

[23] E. Pop, S. Sinha, and K. E. Goodson, "Heat generation and transport in nanometer-scale transistors," Proc. IEEE, vol. 94, no. 8, pp. 1587-1601, Aug. 2006.

[24] M. Gaillardin et al., "Radiation effects in CMOS isolation oxides: Differences and similarities with thermal oxides," IEEE Trans. Nucl. Sci., vol. 60, no. 4, pp. 2623-2629, Aug. 2013.

[25] D. Fleetwood, M. Shaneyfelt, W. Warren, J. Schwank, T. Meisenheimer, and P. Winokur, "Border traps: Issues for MOS radiation response and long-term reliability," Microelectron. Reliab., vol. 35, no. 3, pp. 403-428, 1995.

[26] F. W. Sexton and J. R. Schwank, "Correlation of radiation effects in transistors and integrated circuits," IEEE Trans. Nucl. Sci., vol. NS-32, no. 6, pp. 3975-3981, Dec. 1985.

[27] G. U. Youk, P. S. Khare, R. D. Schrimpf, L. W. Massengill, and K. F. Galloway, "Radiation-enhanced short channel effects due to multidimensional influence from charge at trench isolation oxides," IEEE Trans. Nucl. Sci., vol. 46, no. 6, pp. 1830-1835, Dec. 1999.

[28] A. H. Johnston, R. T. Swimm, G. R. Allen, and T. F. Miyahira, "Total dose effects in CMOS trench isolation regions," IEEE Trans. Nucl. Sci., vol. 56, no. 4, pp. 1941-1949, Aug. 2009.
[29] M. McLain et al., "Enhanced TID susceptibility in sub-100 nm bulk CMOS I/O transistors and circuits," IEEE Trans. Nucl. Sci., vol. 54, no. 6, pp. 2210-2217, Dec. 2007.

[30] A. Czerwinski, E. Simoen, and C. Claeys, "PN junction peripheral current analysis using gated diode measurements," Appl. Phys. Lett., vol. 72 , no. 26 , pp. 3503-3505, 1998 .

[31] Z. Liu et al., "Total ionizing dose enhanced DIBL effect for deep submicron NMOSFET," IEEE Trans. Nucl. Sci., vol. 58, no. 3, pp. 1324-1331, Jun. 2011.

[32] C. H. Wang and P.-F. Zhang, "Three-dimensional DIBL for shallowtrench isolated MOSFET's," IEEE Trans. Electron Devices, vol. 46, no. 1, pp. 139-144, Jan. 1999.

[33] A. J. Lelis, T. R. Oldham, H. E. Boesch, and F. B. McLean, "The nature of the trapped hole annealing process," IEEE Trans. Nucl. Sci., vol. 36 , no. 6, pp. 1808-1815, Dec. 1989.

[34] J. R. Schwank, P. S. Winokur, P. J. McWhorter, F. W. Sexton, P. V. Dressendorfer, and D. C. Turpin, "Physical mechanisms contributing to device "rebound," IEEE Trans. Nucl. Sci., vol. NS-31, no. 6 , pp. 1434-1438, Dec. 1984.

[35] S. N. Rashkeev, D. M. Fleetwood, R. D. Schrimpf, and S. T. Pantelides, "Effects of hydrogen motion on interface trap formation and annealing," IEEE Trans. Nucl. Sci., vol. 51, no. 6, pp. 3158-3165, Dec. 2004.

[36] A. J. Lelis, T. R. Oldham, and W. M. DeLancey, "Response of interface traps during high-temperature anneals," IEEE Trans. Nucl. Sci., vol. 38, no. 6 , pp. 1590-1597, Dec. 1991. 